\title{
Mechanisms of Impulsive Responding to Temporally Predictable Events as Revealed by Electromyography
}

Inga Korolczuk, ${ }^{\mathrm{a}^{*}}$ Boris Burle, ${ }^{\mathrm{b}}$ Jennifer T. Coull ${ }^{\mathrm{b}}$ and Kamila Smigasiewicz $^{\mathrm{b}}$

a Institute of Applied Psychology, Jagiellonian University, ul. Lojasiewicza 4, 30-348 Krakow, Poland

${ }^{\mathrm{b}}$ Laboratoire des Neurosciences Cognitives UMR 7291, Federation 3C, Aix-Marseille University \& CNRS, 3 Place Victor Hugo, 13331 Marseille Cedex 3, France

\section{FUNDING}

This work was supported by the Polish Ministry of Science and Higher Education grant (0050/DIA/2016/45) awarded to IK. The funding source had no impact on any part of the present study. 


\begin{abstract}
Temporal predictability optimises behaviour when a simple response is required, as demonstrated by faster reaction times and higher accuracy. However, its beneficial effects come at cost under situations of response conflict. Here, we investigated the motor underpinnings of behaviour to temporally predictable events in the Simon conflict task. We compared motor responses to lateralised targets whose position conflicted (incompatible condition) or not (compatible condition) with the hand of response. Advanced distributional analyses coupled with electromyographic (EMG) recordings revealed that temporal predictability exaggerated premature impulsive responding as indexed by increased likelihood of fast incorrect EMG activations to incompatible targets, as well as a higher rate and shorter latency of subthreshold EMG activity in the incorrect response agonist preceding a correct response ("partial error”). There was, however, no effect of temporal predictability on subsequent suppression of partial errors. Our results provide direct evidence that temporal predictability acts by increasing the urge to initiate a fast, yet potentially erroneous, response. This mechanism parsimoniously explains both beneficial effects of temporal predictability when no conflict in the environment is present, as well as its costs when more complex motor behaviour is required.
\end{abstract}

Keywords: temporal prediction, timing, motor control, response inhibition, EMG 


\section{Introduction}

Temporal prediction allows us to anticipate and prepare for events that occur at specific moments in time. Indeed, without accurate temporal predictions, we would be unable to accomplish many everyday tasks such as driving a car safely or waiting our turn in conversations. Despite the fact that temporal prediction benefits performance when a simple response is required, for example speeding reaction times (RTs; Correa, Lupiáñez, \& Tudela, 2006; Coull \& Nobre, 1998; Nobre, 2001), reducing muscular effort (Mattes \& Ulrich, 1997; Thomas, French, Alizee, \& Coull, 2019; van der Lubbe, Jaśkowski, \& Verleger, 2004), or improving accuracy (Correa, Lupiáñez, \& Tudela, 2005; Davranche, Nazarian, Vidal, \& Coull, 2011; Martens \& Johnson, 2005; Visser, 2014), its facilitative effects come at cost under more challenging environmental circumstances.

The effects of temporal predictability on potentially competing responses can be studied in conflict tasks (Correa, Cappucci, Nobre, \& Lupiáñez, 2010; Korolczuk, Burle, \& Coull, 2018). In the Simon task (Hommel, 2011; Simon, 1969), for example, participants must provide a lateralised response to a non-spatial stimulus attribute (e.g., if green respond left, if red respond right). The stimulus is, however, presented laterally and, although irrelevant for the task at hand, its position can either match that of the correct response (compatible condition), or the incorrect one (incompatible condition). Typically, performance for incompatible targets is impaired, as demonstrated by slower reaction time (RT) and lower accuracy. Importantly, these global measures of performance can be dissected by advanced distribution analysis methods that reveal conflict resolution dynamics at a finer-grained level. One 
prominent example is the Conditional Accuracy Function (CAF), which shows the probability of correct response as a function of its latency. Crucially, in incompatible conditions, such analyses show that errors mainly occur during very fast responses, but that they are almost non-existent for the slowest responses, revealing a transient susceptibility to execute prepotent, yet potentially incorrect, responses (Ridderinkhof, 2002). The relatively poor accuracy of fast responses to incompatible targets is thought to reflect the strength of automatic response capture and impulsive motor response activation.

Using CAF analysis, we recently demonstrated that anticipating the appearance of a target at a particular moment in time increased the likelihood of fast impulsive errors triggered by the (irrelevant) stimulus position (Korolczuk, Burle, \& Coull, 2018; see also Correa, Cappucci, Nobre, \& Lupiáñez, 2010 for similar pattern of results using flanker and Simon tasks). Yet, this impulsive behaviour induced by temporal predictability could stem from functionally distinct processes: either a stronger temptation to act prematurely or impaired inhibition of premature responses (De Jong, Liang, \& Lauber, 1994; Ridderinkhof, Forstmann, Wylie, Burle, \& van den Wildenberg, 2011; Spieser, van den Wildenberg, Hasbroucq, Ridderinkhof, \& Burle, 2015). The aim of the present study was to elucidate the mechanisms of temporal prediction by dissociating its effects on these two processes.

While much more powerful than standard analyses of mean reaction times, CAF analysis of overt behaviour has two main limitations. First, behaviourally observed errors are only the "tip of the iceberg” of impulsive response capture. Indeed, they reflect only a subsample of incorrect response activations: those that could not be corrected. Second, for this reason, the drop in accuracy observed in CAFs may not 
necessarily indicate stronger prepotent response capture but, instead, an impaired ability to suppress already activated erroneous actions (De Jong et al., 1994). More direct measures are needed to differentiate these possibilities. Notably, recordings of the electromyographic (EMG) activity of muscles involved in response production have proven particularly useful in dissociating the strength of response capture from its subsequent suppression. Recording the EMG reveals that even on correct trials, 15$20 \%$ of all responses to incompatible targets are preceded by a subthreshold burst of EMG activity in the muscle of the incorrect response hand (Burle, Possamaï, Vidal, Bonnet, \& Hasbroucq, 2002; Burle, Spieser, Servant, \& Hasbroucq, 2014; Servant, White, Montagnini, \& Burle, 2015). This phenomenon indexes covert impulsive response activation and is known as a "partial error" to indicate that a potentially erroneous response was corrected before actual response execution. Hence, while EMG allows the expression of activation and suppression mechanisms to be measured at the peripheral level, it also provides information about brain perceptual gating phenomena (Servant, White, Montagnini, \& Burle, 2016) and inhibitory control originating from frontal brain regions (Ficarella, Rochet, \& Burle, 2019) . The rate of partial errors not only provides an index of the susceptibility to response capture, but their latencies can also be used to perform a more dynamic analysis of response activation. In an extension of the classic CAF analysis of overt response accuracy, one can compute the probability that the first EMG activation following a target is produced by the correct or incorrect muscle agonist (irrespective of whether it is actually followed by a correct or incorrect response), as a function of its latency (EMG-CAF). Critically, the EMG-CAF provides a measure of automatic response capture and is independent of any subsequent correction processes; it functionally 
reflects the temporal dynamics of impulse expression directly at the muscular level. In parallel, the measurement of partial errors also allows the efficiency of online suppression of incorrect responses to be assessed. First, the "correction ratio" provides a measure of how often erroneous response impulses are correctly suppressed. It is calculated as (Npe / (Npe + Ner)) where Npe is the number of partial errors and Ner the number of overt errors, and therefore reflects the proportion of corrected incorrect response activations (partial errors) among the overall number of incorrect activations (both partial and overt errors). Second, the correction time (the interval between the incorrect and correct EMG activation, see Figure 2) provides a measure of the time it takes to correct the initial incorrect response activation (Burle et al., 2002). Therefore, while the EMG-CAF provides a direct measure of initial response activation, the correction rate and correction time measure subsequent response suppression. By decoupling these two processes, we recently demonstrated that the higher incidence of fast errors observed in Parkinson's disease patients during deep brain stimulation are not due to increased response capture but, instead reflect decreased efficiency of response suppression (Fluchère et al., 2018)

The goal of the present study was to clarify the motor underpinnings of behaviour to temporally predictable events. Critically, we sought to answer this question by directly studying neurophysiological indices of motor activation and suppression as revealed by electromyography (EMG). Building on previous results indicating that temporal predictability leads to faster response times and an inability to stop prepotent responses (Correa et al., 2010; Korolczuk et al., 2018; although see Correa, Triviño, Pérez-Dueñas, Acosta, \& Lupiáñez, 2010), we aimed to refine the relative contribution of motor activation and inhibition when responding to temporally 
predictable events. In our paradigm, temporal cues predicted the delay between the cue and the target (the "foreperiod" [FP]), which varied within a block. Control "neutral" cues did not provide temporally precise information about the onset of the upcoming target, and so allowed the performance benefits of temporal cueing to be measured. This temporally cued version of the Simon task measures the flexible orientation of attention to particular moments in time and allows us to identify the covert control mechanisms that guide behaviour toward temporally predictable events.

We formulated two alternative hypotheses. First, if the fast impulsive errors induced by temporal predictability are due to an increased urge to emit a premature response, temporal cueing should lead to a higher incidence of incorrect responses to incompatible targets at the fastest EMG latencies. In parallel, no effect of temporal cueing on correction ratio and correction time would be observed. Alternatively, if temporal prediction increases the number of fast errors by impairing response correction processes, the initial part of the EMG-CAF should not be affected (see Fluchère et al., 2018, for an example), and instead, we would expect to see a smaller correction ratio and longer correction times. 


\section{Material and methods}

\subsection{Participants}

Twenty-six healthy participants $\left(M_{\text {age }}=24.6, S D=4.6\right.$; 11 males, 15 females $)$ took part in the study approved by the local research ethics committee. Data from three participants were discarded; one due to technical problems (response triggers not being recorded), one due to high error rate ( $\pm 2 S D$ of the group average) and one due to particularly slow RTs ( $\pm 2 S D$ of the group average). The final sample consisted of 23 participants.

\subsection{Experimental task}

Participants completed a temporally cued version of the Simon task, controlled by PsychoPy software (Peirce et al., 2019). The basic visual display was a black background with central fixation indicated by two concentric white circles $\left(1^{\circ}\right.$ eccentricity). These circles also acted as cues, providing information about the time at which the target would appear. Target stimuli were vertical (“+”) or diagonal (“×”) crosses, sized $1^{\circ}$ visual angle, that appeared either on the left or right side of the central cue at a distance of approximately $3^{\circ}$ of visual angle (Fig. 1).

A cue either predicted (temporal condition) or not (neutral condition) the delay (foreperiod) after which a target would appear. Temporal cues were $100 \%$ valid. In the temporal cue (T) condition, a brightening of the smaller circle informed participants that a target would appear after a short delay (600 ms), whereas brightening of the larger circle indicated that a target would appear after a long delay (1,400 ms). Participants were asked to use the temporal information conveyed by the cues to predict the moment of target appearance. In the neutral cue $(\mathrm{N})$ condition, the entire 
cue brightened, providing no temporally precise information and targets were presented randomly after short or long delays or “foreperiods” (FP). In both conditions, participants were asked to respond with their right or left hand to the shape of the target (“+” or “ $\times$ ”). As each target could be presented on either the left or right side of the screen (target position was irrelevant), the lateralised response required by the target (left/right hand) could be on either the same (compatible condition) or the opposite (incompatible condition) side as the target. The mapping between target and response was counterbalanced across subjects. Participants positioned their thumbs on response buttons placed on two cylindrical handgrips (3 $\mathrm{cm}$ in diameter, $12 \mathrm{~cm}$ in height) fixed vertically to the table (Fig. 1) and pressed one of them with their thumb as quickly as possible after target appearance.

At the beginning of a trial, the cue ( $\mathrm{T}$ or $\mathrm{N}$ ) appeared for $500 \mathrm{~ms}$. The background visual display was then presented for one of the two FPs (short or long). Next, the target appeared for $100 \mathrm{~ms}$ and participants gave their response during a 1,100 ms time window. After the response, the background visual display was again presented on the screen for an inter-trial interval of $900 \mathrm{~ms}-1,400 \mathrm{~ms}$ (with a random jitter of $100 \mathrm{~ms})$.

The two cue ( $\mathrm{T}$ and $\mathrm{N}$ ) conditions were administered six times each in separate blocks. To maintain attentional task set while counterbalancing fatigue/time-on-task effects, three blocks of each cue condition were presented consecutively (TTT-NNNTTT-NNN or NNN-TTT-NNN-TTT). Each block contained 128 trials, resulting in 1,536 trials altogether. In each block, the proportion of compatible to incompatible trials was equal, as was the proportion of short and long FPs, with both factors being 
randomised using Mix software (van Casteren \& Davis, 2006). This resulted in 192 trials for each of the 8 combinations of the cue, FP and compatibility conditions.

Before an experimental session, a training block of 60 trials was provided to familiarise the participants with the task (a series of 5 temporal short trials, followed by a series of 5 temporal long and then 20 mixed temporal short and long trials, and finally a series of 30 neutral trials). Each experimental block lasted approximately 7 minutes, and blocks were separated by short breaks.

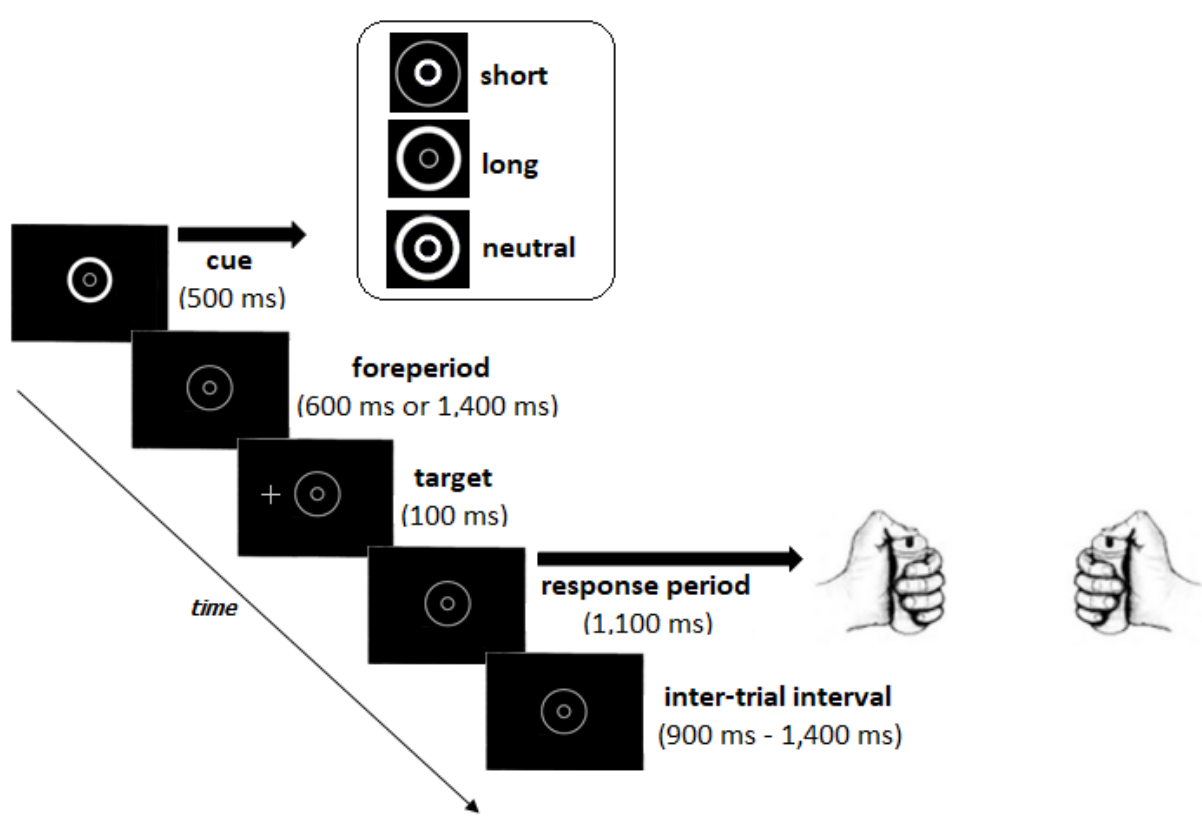

Figure 1. Trial timeline of the task. A cue was presented for 500 ms predicting (or not) the time of target occurrence. A background display was then presented for $600 \mathrm{~ms}$ or 1,400 ms (foreperiod). Next, a target (“+” or “×”) appeared for 100 ms on either the left or right side of the display, followed by a 1,100 ms period of the background display, during which participants made their choice response (left or right thumb for “+” or “×”, counterbalanced across participants). The inter-trial interval was randomised between $900 \mathrm{~ms}$ and 1,400 ms. 


\subsection{Electrophysiological recording and processing}

Bipolar EMG activity of the flexor pollicis brevis from each hand was recorded. Ag/AgCl active flat electrodes (pre-amplified Biosemi Inc., Amsterdam, The Netherlands) were placed about $2 \mathrm{~cm}$ apart on the thenar eminence. EMG activity was digitised online (sample rate: $1,024 \mathrm{~Hz}$, analog bandwidth limit: $-3 \mathrm{~dB}$ at $1 / 5$ th of the sampling rate), with the use of the BioSemi Active-Two system (Biosemi Inc., Amsterdam, The Netherlands) and offline high-pass filtered at $10 \mathrm{~Hz}$. As the quality of the signal is important when detecting the onset of response-related EMG bursts and partial errors, the EMG signal was monitored throughout the experiment and participants were asked to relax their hand muscles if tonic muscle activity became too high.

EMG activity was initially detected using a customised Python script that combines two algorithms, based on a variance comparison (Hodges \& Bui, 1996) and on an “integrated profile” (Liu \& Liu, 2016; Santello \& McDonagh, 1998). To correct for inaccurate detection of EMG bursts by the algorithm, EMG onsets were corrected manually after visual inspection by an experimenter unaware of trial conditions. Based on this procedure, trials were classified as "pure correct” (trials with one EMG burst related to a correct response), "partial errors" (trials with two EMG bursts, the first evoked by an incorrect subthreshold EMG activation, and the second related to a correct response), or “errors” (trials with one EMG burst related to an incorrect response) (Figure 2). 


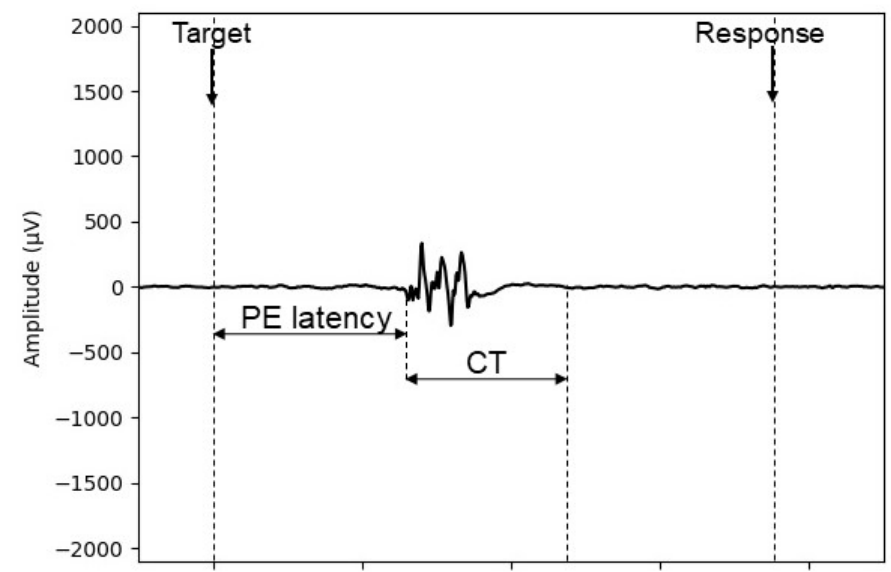

Incorrect EMG

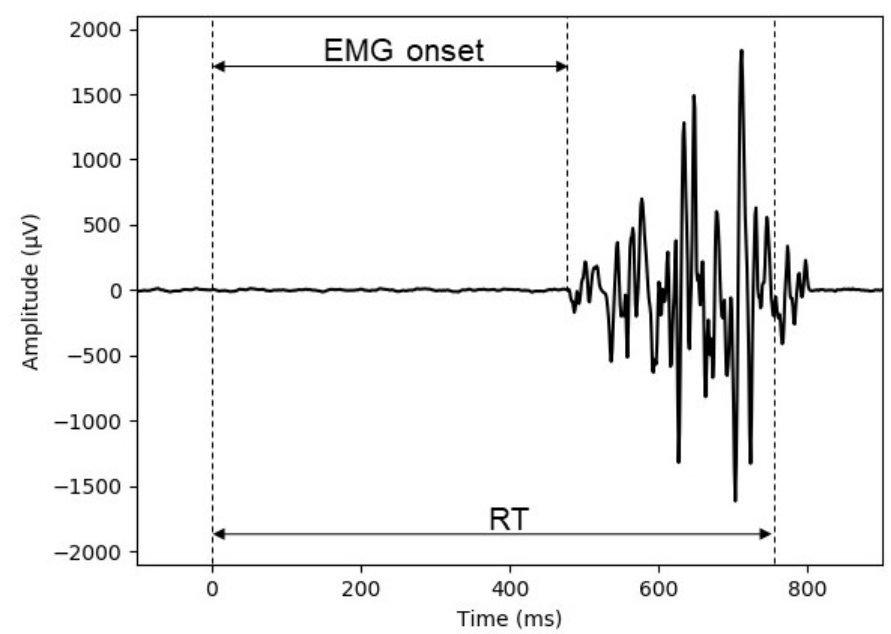

Correct EMG

Figure 2. EMG activity and chronometric measures for a partial error trial.

Subthreshold EMG activity in the incorrect response hand (top panel) is followed by suprathreshold EMG activity in the correct response hand (bottom panel). PE latency denotes partial error latency, CT the correction time and, RT the reaction time.

\section{Data analysis}

\subsubsection{Behavioural measures}

Mean RTs for correct trials were calculated separately for each cue, FP and compatibility condition. Accuracy (\% of overt errors) was calculated as the proportion 
of incorrect responses to all trials (correct, incorrect, missing responses). Missing responses ( 2\%) were not further analysed. The effect of temporal predictability on RTs and accuracy was analysed with a three-way repeated measures ANOVA comprising the following within-subject factors: cue (temporal, neutral), FP (short, long) and compatibility (compatible and incompatible).

To assess dynamic changes in accuracy as a function of RT latencies, Conditional Accuracy Functions (CAF) were computed for each participant and for each of the eight conditions (Cue $\times \mathrm{FP} \times$ Compatibility). CAFs require “vincentisation” (De Jong et al., 1994; Ratcliff, 1979; Vincent, 1912) of the data: after ranking all RTs (from correct and incorrect trials) in ascending order, they were grouped into 5 classes of equal size (quintiles) and the percentage of correct responses within each quintile was quantified. Next, these percentages were entered into a fourway repeated-measures ANOVA with cue (temporal, neutral), FP (short, long), compatibility (compatible, incompatible) and quintile (1 to 5) as within-subject factors.

Bonferroni adjustments were applied for pairwise comparisons in the case of significant interactions. Effect sizes were calculated by using partial eta-squared $\left(\eta_{\mathrm{p}}{ }^{2}\right.$; (Cohen, Cohen, West, \& Aiken, 2003).

\subsubsection{EMG-derived measures}

EMG allowed us to investigate critical chronometric measures (Fig. 2). Partial error rate was calculated as the proportion of all trials containing partial errors. The probability that partial errors would be suppressed was quantified as the Correction Ratio (CR), and defined as the number of partial error trials divided by the overall number of incorrect activation trials (both error and partial error trials). This index 
estimates how often an incorrect response activation was successfully corrected. Furthermore, the response time (RT) in partial error trials was decomposed into a partial error latency (PE; the interval between target onset and onset of a partial error EMG burst) and correction time (CT; the interval between the onset of a partial error and the onset of the correct EMG burst). The effect of temporal predictability on these indices was assessed by a three-way repeated-measures ANOVA involving cue (temporal, neutral), FP (short, long) and compatibility (compatible and incompatible) factors.

To extend the classic CAF analysis of behavioural data, we calculated the probability that the first burst of EMG activity on a given trial was correct as a function of its latency (EMG-CAF). Latencies of correct and incorrect EMG activations (partial and overt errors), calculated as the interval between target onset and first EMG onset, were vincentised into five quantiles. Note that in case of partial error trials, the latency of incorrect EMG activation was the partial error (PE) latency. The effect of temporal predictability on the EMG-CAF was evaluated by a four-way repeated-measures ANOVA with cue (temporal, neutral), FP (short, long), compatibility (compatible, incompatible) and quintile (1 to 5) as within-subject factors. 


\section{Results}

\subsection{Behavioural measures}

\subsubsection{RT and accuracy}

RT and accuracy results are presented in Table 1 . The results showed a main effect of compatibility on RT, $F(1,22)=76.49, p<.001, \eta_{\mathrm{p}}^{2}=.78$. RTs were slower for incompatible than compatible targets. Importantly, there was a main effect of cue, $F(1,22)=5.97, p=.023, \eta_{\mathrm{p}}{ }^{2}=.21$, with participants responding faster in temporally cued trials than in neutral trials. There was no main effect of FP, $F(1,22)=0.32, p=$. $58, \eta_{\mathrm{p}}{ }^{2}=.01$ on RT, or a Cue $\times$ FP interaction, $F(1,22)=3.06, p=.094, \eta_{\mathrm{p}}{ }^{2}=.12$, or a Cue $\times$ Compatibility interaction, $F(1,22)=2.64, p=.119, \eta_{\mathrm{p}}{ }^{2}=.11$, nor FP $\times$ Compatibility interaction, $F(1,22)=1.60, p=.220, \eta_{\mathrm{p}}{ }^{2}=.07$.

The analysis of accuracy (\% overt errors) revealed a main effect of compatibility, $F(1,22)=24.63, p<.001, \eta_{\mathrm{p}}^{2}=.53$. Participants made more errors on incompatible than compatible trials. More errors were also committed in temporal than neutral conditions, $F(1,22)=5.38, p=.030, \eta_{\mathrm{p}}{ }^{2}=.0 .20$. No main effect of FP $F(1,22)=0.03, p=.865, \eta_{\mathrm{p}}^{2}<.01$, was observed.

Table 1

Mean (and standard error) reaction times (ms) and percentage of errors

\begin{tabular}{lllllll}
\hline & & \multicolumn{2}{c}{ Reaction times } & & \multicolumn{2}{c}{$\%$ Errors } \\
\cline { 3 - 4 } \cline { 6 - 7 } Compatibility & FP & Temporal cue & Neutral cue & & Temporal cue & Neutral cue \\
\hline Compatible & Short & $473(12)$ & $491(14)$ & & $3.15(0.70)$ & $2.39(0.48)$ \\
& Long & $479(13)$ & $486(13)$ & & $3.39(0.71)$ & $2.14(0.39)$ \\
Incompatible & Short & $506(13)$ & $518(14)$ & & $6.99(1.17)$ & $5.24(0.98)$ \\
& Long & $506(13)$ & $513(13)$ & & $6.93(1.33)$ & $5.46(0.82)$ \\
\hline
\end{tabular}

\subsubsection{Conditional Accuracy Function}


To explore performance dynamics in more detail, we plotted Conditional Accuracy Functions (CAF). As expected, there were main effects of compatibility, $F(1,22)=19.92, p<.001, \eta_{\mathrm{p}}{ }^{2}=.48$, quintile, $F(4,88)=22.70, p<.001, \eta_{\mathrm{p}}^{2}=.51$ and a Compatibility $\times$ Quintile interaction, $F(4,88)=26.07, p<.001, \eta_{\mathrm{p}}^{2}=.54$. Planned comparisons revealed that accuracy was lower for incompatible than compatible targets only in quintile $1(p<.001)$, which comprised the trials with the fastest RTs. There was a significant main effect of cue, $F(1,22)=7.21, p=.013, \eta_{\mathrm{p}}{ }^{2}=.20$, which was further qualified by a significant Cue $\times$ Compatibility $\times$ Quintile interaction, $F(4$, 88) $=2.53, p=.046, \eta_{\mathrm{p}}{ }^{2}=.10$. For the fastest RTs (quintile 1 ), temporal predictability induced significantly more errors to incompatible targets than when no temporally precise information was present, $p<.001$ (Fig. 3). These results replicate our previous findings (Korolczuk et al., 2018). 


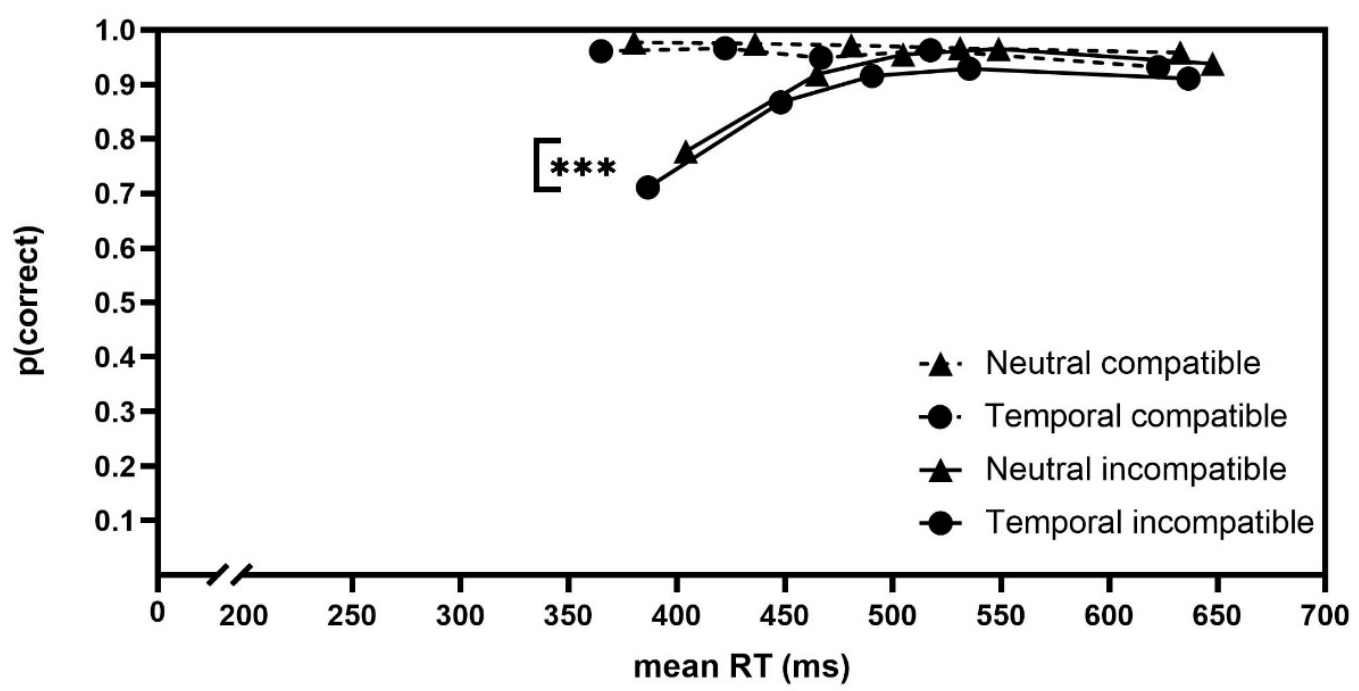

Figure 3. Conditional Accuracy Function (CAF). The plots show the probability of a correct response as a function of RT for both cue (temporal, neutral) and compatibility (compatible, incompatible) conditions. Participants made more fast errors to incompatible targets after temporal cues (circle) than after neutral cues (triangle), indicating stronger response capture when events were temporally predictable.

\subsection{EMG measures}

\subsubsection{Partial error rate and latency}

As expected, results showed a main effect of compatibility on partial error rate, $\left.F(1,22)=47.12, p<.001, \eta_{\mathrm{p}}^{2}=.68\right)$. Incorrect subthreshold bursts of EMG activity (subsequently corrected) were more frequent in incompatible than compatible trials. Importantly, partial errors were also more frequent after temporal than neutral cues, $F(1,22)=19.46, p<.001, \eta_{\mathrm{p}}{ }^{2}=.47$. In line with these findings, the analysis of partial error latency revealed that partial errors were made earlier during temporal than neutral trials, $F(1,22)=11.33, p=.003, \eta_{\mathrm{p}}{ }^{2}=.34$. Finally, partial errors were also faster during incompatible than compatible trials, $F(1,22)=56.49, p<.001, \eta_{\mathrm{p}}{ }^{2}$ 
$=.72$ (replicating previous results, see for example Burle et al., 2002) , and after long than short FPs, $F(1,22)=9.37, p=.006, \eta_{\mathrm{p}}^{2}=.30$.

Table 2

Mean (and standard error) partial error latency (ms) and percentage of partial errors

\begin{tabular}{lllllll}
\hline & & \multicolumn{2}{c}{ Partial error latencies } & & \multicolumn{2}{c}{ \% Partial errors } \\
\cline { 3 - 4 } \cline { 6 - 7 } Compatibility & FP & Temporal cue & Neutral cue & & Temporal cue & Neutral cue \\
\hline Compatible & Short & $319(11)$ & $326(10)$ & & $12.4(1.46)$ & $10.0(1.01)$ \\
& Long & $309(9)$ & $316(11)$ & & $12.0(1.31)$ & $9.20(1.08)$ \\
Incompatible & Short & $277(9)$ & $297(10)$ & & $27.1(2.66)$ & $23.6(2.93)$ \\
& Long & $273(9)$ & $278(9)$ & & $27.4(3.15)$ & $24.8(2.97)$ \\
\hline
\end{tabular}

\subsubsection{EMG-Conditional Accuracy Function}

The analysis of EMG-CAF revealed that for the fastest EMG latencies approximately $76 \%$ of all initial EMG bursts of activity to incompatible targets were incorrect (Fig. 4), replicating previous findings (e.g., Fluchère et al., 2018; van den Wildenberg et al., 2010). Again, there was a main effect of compatibility, $F(1,22)=$ 53.17, $p<.001, \eta_{\mathrm{p}}{ }^{2}=.71$, quintile, $F(4,88)=146.03, p<.001, \eta_{\mathrm{p}}{ }^{2}=.87$, and a Compatibility $\times$ Quintile interaction, $F(4,88)=74.52, p<.001, \eta_{\mathrm{p}}{ }^{2}=.77$. Accuracy was lower for incompatible than compatible targets only in quintiles $1(p<.001)$ and $2(p<.001)$. Importantly, a main effect of cue, $F(1,22)=18.96, p<.001, \eta_{p}{ }^{2}=.46$, was qualified by a Cue $\times$ Compatibility $\times$ Quintile interaction, $F(4,88)=2.42, p=$. $054, \eta_{\mathrm{p}}{ }^{2}=.10$. Temporally predictable targets induced incorrect EMG activation on $80 \%$ of the fastest EMG latency trials, as compared to $72 \%$ in the neutral condition ( $p$ $=.027)$. 


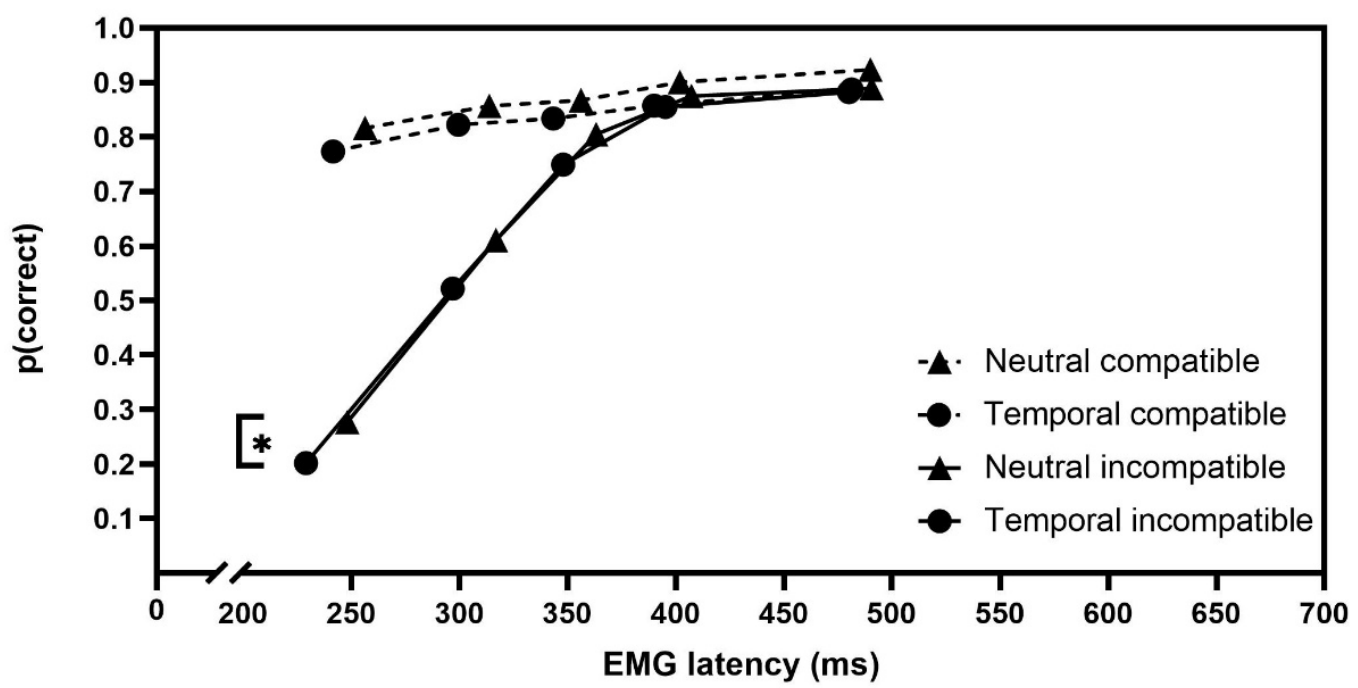

\subsubsection{Correction ratio and time}

Figure 4. Conditional Accuracy Function (CAF) based on EMG data. The plots show the probability of correct muscle activation as a function of EMG latency for both cue (temporal, neutral) and compatibility (compatible, incompatible) conditions. In the temporal condition (circles), 80\% of the fastest muscle activations to incompatible targets were incorrect as compared to $72 \%$ in the neutral condition (triangles).

Temporal predictability therefore increased motor activation of incorrect prepotent responses.

Crucially, there was no main effect of cue on correction ratio, $F(1,22)=0.38, p$ $=.542, \eta_{\mathrm{p}}{ }^{2}=.02$, or a Cue $\times$ Compatibility interaction, $F(1,22)=0.01, p=.931, \eta_{\mathrm{p}}{ }^{2}$ $<.001$, indicating that the increased number of overt errors after temporal cues were not due to diminished ability to suppress an activated prepotent response. All other main effects and interactions in the analysis of correction ratio were also nonsignificant.

Correction time was faster in compatible than incompatible trials, $F(1,22)=$ 23.40, $p<.001, \eta_{\mathrm{p}}{ }^{2}=.52$, replicating previous reports (Burle et al., 2002; Fluchère et 
al., 2018). We also found a significant FP $\times$ Compatibility interaction, $F(1,22)=5.42$, $p=.029, \eta_{\mathrm{p}}^{2}=.20$. In the incompatible condition, correction time was faster after short than long FPs ( $p=.066)$, independently of the nature of the cue. However, in compatible trials there was no difference in correction time between short and long FP trials $(p=1.00)$. Importantly, no main effect of cue $F(1,22)=0.38, p=.545, \eta_{\mathrm{p}}{ }^{2}=$. 02 , or a Cue $\times$ Compatibility interaction, $F(1,22)=2.76, p=.11, \eta_{\mathrm{p}}{ }^{2}=.11$, was found. Similarly, no main effect of $\mathrm{FP}, F(1,22)=1.94, p=.177, \eta_{\mathrm{p}}{ }^{2}=.08$, or a Cue $\times$ FP interaction, $F(1,22)=1.30, p=.266, \eta_{\mathrm{p}}{ }^{2}=.06$, was observed.

Table 3

Mean (and standard error) correction time (ms) and ratio

\begin{tabular}{lllllll}
\hline & & \multicolumn{2}{c}{ Correction time } & & \multicolumn{2}{c}{ Correction ratio (\%) } \\
\cline { 3 - 4 } \cline { 6 - 7 } Compatibility & FP & Temporal cue & Neutral cue & & Temporal cue & Neutral cue \\
\hline Compatible & Short & $158(7)$ & $153(9)$ & & $80.9(3.4)$ & $81.7(2.8)$ \\
& Long & $149(8)$ & $156(9)$ & & $79.6(3.3)$ & $80.9(3.5)$ \\
Incompatible & Short & $175(8)$ & $165(9)$ & & $79.5(2.9)$ & $80.9(2.7)$ \\
& Long & $181(7)$ & $178(6)$ & & $80.2(3.0)$ & $80.5(2.5)$ \\
\hline
\end{tabular}

\section{Discussion}

Performance benefits of temporal prediction have consistently been demonstrated in simple RT detection tasks. Although speeded RTs and improved accuracy have been linked to enhanced perceptual processing (Correa, Lupiáñez, \& Tudela, 2005; Rolke \& Hofmann, 2007; Vangkilde, Coull, \& Bundesen, 2012), improved response preparation (Correa \& Nobre, 2008; Miniussi, Wilding, Coull, \& Nobre, 1999; Nobre, 2001) and reduced muscular effort (Mattes \& Ulrich, 1997; Thomas et al., 2019; van der Lubbe et al., 2004), most studies on temporal predictability have investigated responses based on noncompeting stimulus-action representations. We recently 
demonstrated (Korolczuk et al., 2018) that temporal predictability in fact yielded detrimental effects in conflict situations by increasing fast impulsive responses to incompatible targets. In the current experiment, we sought to better characterise the effects of temporal prediction on motor control mechanisms during response conflict by employing EMG recordings coupled with distribution analysis. This methodology allowed to look beyond overt errors and study covert impulsive response activation and its subsequent suppression.

Overall, behavioural data showed that participants were faster and made more errors after temporal cueing. We further explored these effects by plotting accuracy as a function of RT. This CAF analysis revealed that for the fastest RTs, there were more errors to incompatible targets after temporal than neutral cues. This pattern replicates our previous results, with temporal prediction exaggerating the tendency to produce fast erroneous responses (Korolczuk et al., 2018). Therefore, behavioural data confirmed the detrimental effects of temporal predictability in situations of response conflict.

To reveal the mechanisms of impulsive responding induced by temporal predictability, we then turned to EMG activity induced by correct or incorrect responses. The EMG results revealed stronger response capture in temporally cued trials as demonstrated by more frequent partial errors, which index covert impulsive activations (Spieser et al., 2015; van den Wildenberg et al., 2010). Moreover, these partial errors appeared more quickly after temporal than neutral cues, indicating an overall effect of temporal cueing on premature response activation. To directly assess the relative strength of the tendency to react impulsively from a subsequent ability to suppress this impulse, we looked at the EMG-CAF. This analysis revealed that $76 \%$ of 
the fastest muscular activations to incompatible targets were incorrect. Importantly, the EMG-CAF data further revealed that temporal predictability provoked a higher number of fast incorrect muscle activations to incompatible targets, thus providing further evidence that temporal cueing increases impulsive responding. By contrast, our analyses revealed no effect of temporal prediction on response correction, as indexed by the correction ratio and correction time. Overall, our findings suggest that temporally predictable targets make participants more vulnerable to response capture by prepotent stimulus-response associations, thereby inducing a higher proportion of inappropriate responses (committed overtly or covertly). In other words, temporal predictability increases the tendency to act prematurely but does not impair subsequent suppression of these incorrect impulses.

Our results also further validate electromyography as a sensitive method for studying motor control processes not otherwise easily detected by classical measures. For example, while previous studies have found effects of experimental manipulation selectively on suppression processes (e.g., Fluchère et al., 2018), our data indicate a selective effect on response capture. This dissociation further supports a theoretical dissociation between these two independent mechanisms.

Our previous findings indicate that having a temporal expectation for the time of target appearance makes it harder to stop a response to the target (Korolczuk et al., 2018), suggesting that temporal cueing affects response inhibition. The present results, which indicate an effect of temporal predictability on response activation rather than inhibition, might therefore seem contradictory. However, it is important to dissociate the effects of temporal prediction on selective inhibitory processes (i.e., inhibiting one response in favour of another) versus global inhibition (i.e., 
withholding the response entirely). Indeed, we found that although temporal predictability impairs the ability to inhibit action in the Stop-signal task, the effectiveness of response suppression in the Simon task was unaffected. This dissociation suggests that knowing when a target will occur makes it harder to stop a response completely (“should I make a response”?) but does not affect resolution of conflicting responses (“which response should I make”?). Nevertheless, although both tasks probe the ability to suppress an action (selective or global), the strength of the relative tendency to act not only depends on inhibition, but also on response activation. Even for the same levels of inhibitory strength, the more strongly activated response will be harder to stop. Thus, impaired stopping of responses to temporally predictable events can also result from stronger response activation.

Collectively, our data suggest that temporal predictability increases the overall level of motor activation. This pattern of results is in line with functional brain imaging data showing increased functional connectivity between left intraparietal sulcus (IPS), a region consistently linked to the orienting of attention to temporally predictable targets (Coull \& Nobre, 1998; Coull, Davranche, Nazarian, \& Vidal, 2013), and premotor/motor cortices (Davranche et al., 2011). One may hypothesise that when preparing to make a motor response at a predictable moment in time, a topdown modulatory signal is sent from left IPS to motor cortex. By analogy with the biased competition model of spatial attention (Desimone \& Duncan, 1995; Kastner \& Ungerleider, 2001), this increase in motor cortex activity might serve to prioritise motor processing at that specific moment in time. Importantly, a recent electrophysiological study further confirmed the effect of temporal expectations on preparatory motor activation as revealed by modulation of premotor potentials by 
temporally predictable versus unpredictable targets, accompanied by a decrease in central alpha power (Volberg \& Thomaschke, 2017). Although beneficial for simple actions, this built up of motor activation might prove detrimental when anticipating events calling for more complex or conflicting responses.

To conclude, our study aimed to identify the motor control mechanisms that induce impulsive responding to temporally predictable events. Using direct electromyographic measures and distributional analyses, we were able to separate impulsive motor activation from its subsequent inhibition. Our results indicate that temporal prediction acts to exacerbate the impulse to response prematurely. Conversely, directing attentional resources to particular moments in time does not affect corrective inhibitory processes. These results recast the documented enhancements in performance that have been reported when participants prepare to act at an expected time. Whereas temporal attention generally speeds responses, its effects might be detrimental when more complex, or competing, responses are required. This mechanism of temporal prediction is pertinent for a wide variety of cognitive fields, whenever participants must react after a temporally predictable (e.g., fixed) interval. 
Declarations of interest: none

Funding: This work was supported by the Polish Ministry of Science and Higher Education grant (0050/DIA/2016/45) awarded to IK. The funding source had no impact on any part of the present study. 


\section{References}

Burle, B., Possamaï, C.-A., Vidal, F., Bonnet, M., \& Hasbroucq, T. (2002). Executive control in the Simon effect: an electromyographic and distributional analysis. Psychological Research, 66(4), 324-336. https://doi.org/10.1007/s00426-0020105-6

Burle, B., Spieser, L., Servant, M., \& Hasbroucq, T. (2014). Distributional reaction time properties in the Eriksen task: Marked differences or hidden similarities with the Simon task? Psychonomic Bulletin and Review, 21(4), 1003-1010. https://doi.org/10.3758/s13423-013-0561-6

Cohen, J., Cohen, P., West, S. G., \& Aiken, L. S. (2013). Applied multiple regression/correlation analysis for the behavioral Sciences: Taylor \& Francis. https://psycnet.apa.org/record/2002-18109-000

Correa, Á., Cappucci, P., Nobre, A. C., \& Lupiáñez, J. (2010). The two sides of temporal orienting: Facilitating perceptual selection, disrupting response selection. Experimental Psychology, 57(2), 142-148. https://doi.org/10.1027/1618-3169/a000018

Correa, Á., Lupiáñez, J., \& Tudela, P. (2005). Attentional preparation based on temporal expectancy modulates processing at the perceptual level. Psychonomic Bulletin and Review, 12(2), 328-334. https://doi.org/10.3758/BF03196380

Correa, Á., Lupiáñez, J., \& Tudela, P. (2006). The attentional mechanism of temporal orienting: Determinants and attributes. Experimental Brain Research, 169(1), 58-68. https://doi.org/10.1007/s00221-005-0131-x

Correa, A., \& Nobre, A. C. (2008). Neural modulation by regularity and passage of time. Journal of Neurophysiology, 100(3), 1649-1655. 
https://doi.org/10.1152/jn.90656.2008

Correa, Á., Triviño, M., Pérez-Dueñas, C., Acosta, A., \& Lupiáñez, J. (2010).

Temporal preparation, response inhibition and impulsivity. Brain and Cognition, 73(3), 222-228. https://doi.org/10.1016/j.bandc.2010.05.006

Correa, Angel, Lupiáñez, J., \& Tudela, P. (2005). Attentional preparation based on temporal expectancy modulates processing at the perceptual level. Psychonomic Bulletin \& Review, 12(2), 328-334. https://doi.org/10.3758/BF03196380

Coull, J. T., Davranche, K., Nazarian, B., \& Vidal, F. (2013). Functional anatomy of timing differs for production versus prediction of time intervals.

Neuropsychologia, 51(2), 309-319.

https://doi.org/10.1016/j.neuropsychologia.2012.08.017

Coull, J. T., \& Nobre, A. C. (1998). Where and when to pay attention: The neural systems for directing attention to spatial Locations and to time intervals as revealed by both PET and fMRI. The Journal of Neuroscience, 18(18), 74267435. https://doi.org/10.1523/jneurosci.18-18-07426.1998

Davranche, K., Nazarian, B., Vidal, F., \& Coull, J. (2011). Orienting attention in time activates left intraparietal sulcus for both perceptual and motor task goals. Journal of Cognitive Neuroscience, 23(11), 3318-3330. https://doi.org/10.1162/jocn_a_00030

De Jong, R., Liang, C. C., \& Lauber, E. (1994). Conditional and unconditional automaticity: A dual-process model of effects of spatial stimulus-response correspondence. Journal of Experimental Psychology: Human Perception and Performance, 20(4), 731-750. https://doi.org/10.1037/0096-1523.20.4.731

Desimone, R., \& Duncan, J. (1995). Neural mechanisms of selective visual attention. 
Annual Review of Neuroscience, 18(1), 193-222.

https://doi.org/10.1146/annurev.ne.18.030195.001205

Ficarella, S. C., Rochet, N., \& Burle, B. (2019). Becoming aware of subliminal responses: an EEG/EMG study on partial error detection and correction in humans. Cortex. https://doi.org/10.1016/J.CORTEX.2019.07.007

Fluchère, F., Burle, B., Vidal, F., van den Wildenberg, W., Witjas, T., Eusebio, A., ... Hasbroucq, T. (2018). Subthalamic nucleus stimulation, dopaminergic treatment and impulsivity in Parkinson's disease. Neuropsychologia, 117, 167-177. https://doi.org/10.1016/j.neuropsychologia.2018.02.016

Hasbroucq, T., Osman, A., Possamaï, C.-A., Burle, B., Carron, S., Dépy, D., ... Mouret, I. (1999). Cortico-spinal inhibition reflects time but not event preparation: neural mechanisms of preparation dissociated by transcranial magnetic stimulation. Acta Psychologica, 101(2-3), 243-266. https://doi.org/10.1016/S0001-6918(99)00007-4

Hodges, P. W., \& Bui, B. H. (1996). A comparison of computer-based methods for the determination of onset of muscle contraction using electromyography. Electroencephalography and Clinical Neurophysiology, 101(6), 511-519. https://doi.org/10.1016/s0013-4694(96)95190-5

Hommel, B. (2011). The Simon effect as tool and heuristic. Acta Psychologica, 136(2), 189-202. https://doi.org/10.1016/J.ACTPSY.2010.04.011

Kastner, S., \& Ungerleider, L. G. (2001). The neural basis of biased competition in human visual cortex. Neuropsychologia, 39(12), 1263-1276. https://doi.org/10.1016/S0028-3932(01)00116-6

Korolczuk, I., Burle, B., \& Coull, J. T. (2018). The costs and benefits of temporal 
predictability: impaired inhibition of prepotent responses accompanies increased activation of task-relevant responses. Cognition, 179, 102-110. https://doi.org/10.1016/j.cognition.2018.06.006

Liu, J., \& Liu, Q. (2016). Use of the integrated profile for voluntary muscle activity detection using EMG signals with spurious background spikes: A study with incomplete spinal cord injury. Biomedical Signal Processing and Control, 24, 19-24. https://doi.org/10.1016/J.BSPC.2015.09.004

Martens, S., \& Johnson, A. (2005). Timing attention: Cuing target onset interval attentional blink. Memory \& Cognition, 33(2), 234-240. https://doi.org/10.3758/bf03195312

Mattes, S., \& Ulrich, R. (1997). Response force is sensitive to the temporal uncertainty of response stimuli. Perception \& Psychophysics, 59(7), 1089-1097. https://doi.org/10.3758/BF03205523

Miniussi, C., Wilding, E. L., Coull, J. T., \& Nobre, A. C. (1999). Orienting attention in time. Modulation of brain potentials. Brain, 122(8), 1507-1518. https://doi.org/10.1093/brain/122.8.1507

Nobre, A. C. (2001). Orienting attention to instants in time. Neuropsychologia, 39(12), 1317 - 1328. https://doi.org/10.1016/S0028-3932(01)00120-8

Peirce, J., Gray, J. R., Simpson, S., MacAskill, M., Höchenberger, R., Sogo, H., ... Lindeløv, J. K. (2019). PsychoPy2: Experiments in behavior made easy. Behavior Research Methods, 51(1), 195-203. https://doi.org/10.3758/s13428018-01193-y

Ratcliff, R. (1979). Group reaction time distributions and an analysis of distribution statistics. Psychological Bulletin, 86(3), 446-461. 
http://psycnet.apa.org/fulltext/1979-27730-001.pdf

Ridderinkhof, K. R. (2002). Activation and suppression in conflict tasks: empirical clarification through distributional analyses. Common Mechanisms in Perception and Action, 68335, 26.

http://onlinelibrary.wiley.com/doi/10.1002/cbdv.200490137/abstract

Ridderinkhof, R. K., Forstmann, B. U., Wylie, S. A., Burle, B., \& van den Wildenberg, W. P. M. (2011). Neurocognitive mechanisms of action control: Resisting the call of the Sirens. Wiley Interdisciplinary Reviews: Cognitive Science, 2(2), 174-192. https://doi.org/10.1002/wcs.99

Rolke, B., \& Hofmann, P. (2007). Temporal uncertainty degrades perceptual processing. Psychonomic Bulletin and Review, 14(3), 522-526. https://doi.org/10.3758/BF03194101

Santello, M., \& McDonagh, M. J. (1998). The control of timing and amplitude of EMG activity in landing movements in humans. Experimental Physiology, 83(6), 857-874. https://doi.org/10.1113/expphysiol.1998.sp004165

Servant, M., White, C., Montagnini, A., \& Burle, B. (2015). Using covert response activation to test latent assumptions of formal decision-making models in humans. Journal of Neuroscience, 35(28), 10371-10385. https://doi.org/10.1523/JNEUROSCI.0078-15.2015

Servant, Mathieu, White, C., Montagnini, A., \& Burle, B. (2016). Linking theoretical decision-making mechanisms in the Simon task with electrophysiological data: A model-based neuroscience study in humans. Journal of Cognitive Neuroscience, 28(10), 1501-1521. https://doi.org/10.1162/jocn_a_00989

Simon, J. R. (1969). Reactions toward the source of stimulation. Journal of 
Experimental Psychology, 81(1), 174-176. http://dx.doi.org/10.1037/h0027448

Spieser, L., van den Wildenberg, W., Hasbroucq, T., Ridderinkhof, K. R., \& Burle, B. (2015). Controlling your impulses: Electrical stimulation of the human supplementary motor complex prevents impulsive errors. Journal of Neuroscience, 35(7), 3010-3015. https://doi.org/10.1523/JNEUROSCI.164214.2015

Thomas, E., French, R., Alizee, G., \& Coull, J. T. (2019). Having your cake and eating it: Faster responses with reduced muscular activation while learning a temporal interval. Neuroscience, 410, 68-75. https://doi.org/10.1016/J.NEUROSCIENCE.2019.05.003

van Casteren, M., \& Davis, M. H. (2006). Mix, a program for pseudorandomization. Behavior Research Methods, 38(4), 584-589. https://doi.org/10.3758/BF03193889

van den Wildenberg, W. P. M., Wylie, S. A., Forstmann, B. U., Burle, B., Hasbroucq, T., \& Ridderinkhof, K. R. (2010). To head or to heed? Beyond the surface of selective action inhibition: A Review. Frontiers in Human Neuroscience, 4. https://doi.org/10.3389/fnhum.2010.00222

van der Lubbe, R. H. J., Los, S. A., Jaśkowski, P., \& Verleger, R. (2004). Being prepared on time: On the importance of the previous foreperiod to current preparation, as reflected in speed, force and preparation-related brain potentials. Acta Psychologica, 116(3), 245-262. https://doi.org/10.1016/j.actpsy.2004.03.003

Vangkilde, S., Coull, J. T., \& Bundesen, C. (2012). Great expectations: Temporal expectation modulates perceptual processing speed. Journal of Experimental 
Psychology: Human Perception and Performance, 38(5), 1183-1191. https://doi.org/10.1037/a0026343

Vincent, S.B. (1912). The function of vibrissae in the behavior of the white rat. Behavior Monographs, 1(5), 1-82.

Visser, T. A. W. (2014). Evidence for deficits in the temporal attention span of poor readers. PLoS ONE, 9(3). https://doi.org/10.1371/journal.pone.0091278

Volberg, G., \& Thomaschke, R. (2017). Time-based expectations entail preparatory motor activity. Cortex, 92, 261-270. https://doi.org/10.1016/j.cortex.2017.04.019 